\title{
Consumo de bebidas alcoólicas e prática do binge drinking entre cabeleireiros
}

\section{Consumption of alcoholic beverages and practice of binge drinking among hairdressers}

\author{
Como citar este artigo:
}

Castelo Branco FMF, Sousa GT, Marques NQS, Moraes AAS, Trindade FR, Branco Neto TC, et al. Consumption of alcoholic beverages and practice of binge drinking among hairdressers. Rev Rene. 2021;22:e60574. DOI: https://doi.org/10.15253/2175-6783.20212260574
${ }^{1}$ Universidade Federal do Amapá.

Oiapoque, AP, Brasil.

${ }^{2}$ Universidade Federal do Piauí.

Teresina, PI, Brasil.

\section{Autor correspondente:}

Fernanda Matos Fernandes Castelo Branco

Rodovia BR 156, 3051, Bairro Universidade,

CEP: 68980-000. Oiapoque, AP, Brasil.

E-mail: fmfernandescb@gmail.com
EDITOR CHEFE: Ana Fatima Carvalho Fernandes EDITOR ASSOCIADO: Renan Alves Silva

\begin{abstract}
RESUMO
Objetivo: verificar o consumo de bebidas alcóolicas e a prática do binge drinking entre os cabeleireiros. Métodos: estudo transversal realizado com 51 profissionais de salões de beleza. Utilizou-se de um questionário com características sociodemográficas e sobre as práticas do consumo de bebidas alcoólicas. Para a identificação do uso em binge drinking, pautou-se a questão-chave. Realizou-se a análise estatística descritiva e inferencial. Resultados: $84,3 \%$ eram consumidores de álcool, 51,0\% tinham de um a dez anos de consumo e $72,5 \%$ consumiam a cerveja. Em relação ao uso em binge, $37,3 \%$ da amostra faziam uso ocasional de risco, pelo menos, uma vez ao mês. Os maiores índices de binge drinking estavam relacionados ao gênero masculino, aos solteiros e jovens e a religião evangélica foi associada a um menor ou nenhum consumo de bebidas alcoólicas. Conclusão: os dados apontaram o consumo alcoólico e o uso em binge frequente relacionados à provável dependência alcoólica.

Descritores: Alcoolismo; Bebedeira; Centros de Embelezamento e Estética; Transtornos Induzidos por Álcool; Transtornos Relacionados ao Uso de Substâncias.
\end{abstract}

\section{ABSTRACT}

Objective: to verify the consumption of alcoholic beverages and the practice of binge drinking among hairdressers. Methods: cross-sectional study conducted with 51 beauty salon professionals. We used a questionnaire with socio-demographic characteristics and about the practices of consumption of alcoholic beverages. For the identification of the use in binge drinking, the key question was guided. Descriptive and inferential statistical analysis was performed. Results: $84.3 \%$ were alcohol consumers, $51.0 \%$ had between one and ten years of consumption and $72.5 \%$ consumed beer. In relation to the use in binging, $37.3 \%$ of the sample made occasional use of risk, at least once a month. The highest rates of binge drinking were related to male gender, single and young and the evangelical religion was associated with a lower or no consumption of alcoholic beverages. Conclusion: the data pointed to alcohol consumption and frequent binge use related to likely alcohol dependence.

Descriptors: Alcoholism; Binge Drinking; Beauty and Aesthetics Centers; Alcohol-Induced Disorders; Substance-Related Disorders. 


\section{Introdução}

Dentre os padrões de consumo, destaca-se o beber pesado episódico (heavy episodic drinking) ou outros padrões de consumo do álcool, como beber pesado (heavy drinking), também conhecido como uso ocasional de risco, internacionalmente denominado de binge drinking, que se caracteriza pelo consumo de quatro doses ou mais para pessoas do gênero feminino e cinco doses ou mais para o gênero masculino na mesma ocasião. Enfatiza-se que a dose padrão contém, aproximadamente, de dez a $12 \mathrm{~g}$ de álcool puro, equivalendo a uma lata de cerveja $(330 \mathrm{ml})$, dose de destilado $(30 \mathrm{ml})$ ou taça de vinho $(100 \mathrm{ml})$. Entretanto, tal definição é refutável por tal conceito poder sofrer influência dos aspectos culturais e farmacocinéticos do álcool ${ }^{(1)}$.

Assim, ao avaliar a tendência do consumo abusivo de álcool, conforme a Organização Mundial de Saúde (OMS), notou-se uma diminuição da frequência do beber pesado episódico em nível global, pois, no ano 2000 , correspondia a $22,6 \%$ e, em 2016, passou para 18,2\% na população acima de 15 anos. No Brasil, esse padrão aumentou de $12,7 \%$, no ano de 2010 , para $19,4 \%$, em $2016^{(2)}$.

Ainda no concernente ao território brasileiro, ao analisar a tendência de consumo entre os anos de 2006 e 2013, a Vigilância de Fatores de Risco e Proteção para Doenças Crônicas por Inquérito Telefônico, que avaliou a população adulta acima de 18 anos, mostrou que $15,6 \%$, em 2006, e 16,4\%, em 2013, faziam uso ocasional de risco $^{(3)}$. De acordo com o III Levantamento Nacional sobre o Uso de Drogas, o uso em binge foi de 16,5\%, correspondendo a 25 milhões de habitantes da população geral. Isto posto, o estudo instilou a mudança significativa dos padrões de consumo de álcool em território brasileiro ${ }^{(4)}$.

Diante dessas circunstâncias e refletindo acerca da parcela significativa da classe adulta e trabalhadora, destacam-se, nesse segmento, os (as) cabeleireiros (as), trabalhadores que possuem carga horária de trabalho exaustiva, pois, devido ao aumento do poder aquisitivo da população e à influência dos meios de comunicação, se tem inspirado os seres humanos a se preocuparem com a beleza, cuidados com o corpo e, assim, são necessários os profissionais de beleza para atender às demandas ${ }^{(5)}$. Desse modo, no município de Oiapoque, a maioria deles está localizada no centro da cidade, com fácil acesso aos bares e pontos de vendas de bebidas alcóolicas, favorecendo o consumo dessas substâncias, sendo, muitas vezes, esse uso de risco.

Desse modo, ao considerar a ausência de estudos na literatura brasileira acerca do consumo de álcool e da prática do binge drinking entre os (as) cabeleireiros (as) de centros de embelezamento e estética, bem como a inexistência de dados na cidade de Oiapoque junto a essa classe profissional, que apresenta peculiaridades relacionadas às condições laborais, como excesso na carga horária de trabalho, crescimento dessa classe trabalhadora sem qualificação profissional, exposição de clientes e trabalhadores a riscos ocupacionais, incluindo doenças infectocontagiosas ${ }^{(5)}$, além da carência de assistência à saúde nesses trabalhadores, surgiu a necessidade de preencher lacunas acerca do tema, buscando a disseminação do desenvolvimento de estratégias de prevenção acerca do uso problemático de álcool junto ao público investigado.

Assim, a formação de profissionais de saúde, especialmente os enfermeiros, é essencial para a detecção precoce dos consumos de risco de álcool, além da implementação de técnicas que visam à redução do consumo e à intervenção nos casos identificados, além de estratégias e intervenções mais condizentes com usuários de álcool para que, dessa forma, se desenvolvam programas de atendimento e políticas de saúde eficazes $^{(6)}$.

Logo, objetivou-se verificar o consumo de bebidas alcóolicas e a prática do binge drinking entre os cabeleireiros

\section{Métodos}

Estudo transversal, com amostra não probabilística, realizado em 31 salões de beleza. Destaca- 
-se que, em cada estabelecimento, havia de um a três profissionais. Pela particularidade do município, não existem centros de embelezamento e estética de grande porte e essa classe trabalhista não contava com entidade, associação ou órgão competente que os representasse, portanto, não foi possível estimar a quantidade exata de pessoas de tal profissão em Oiapoque. Por isso, buscou-se pelos que estavam no centro da cidade por meio da técnica Snowball Sampling.

Os participantes iniciais do estudo indicaram novos participantes que, por sua vez, designavam novos possíveis participantes e, assim, sucessivamente, até se alcançar o objetivo proposto. Portanto, adotou-se a técnica de amostragem, que se utilizou das cadeias de referência. Dessa forma, localizavam-se os informantes-chave, nos salões situados na zona central, que nomeavam os mais afastados e, na sequência, as visitas foram realizadas aos salões localizados no extremo norte do Amapá, Brasil, na fronteira franco-brasileira, de julho de 2018 a junho de 2019.

Entre os critérios de inclusão, estabeleceram-se: profissionais acima de dezoito anos e de ambos os gêneros, que trabalhassem há mais de um ano em salões de beleza. Excluíram-se da amostra indivíduos que, no momento da coleta, apresentaram alterações de comportamentos visíveis ou estavam intoxicados.

Para a coleta de dados, os pesquisadores contataram diretamente os cabeleireiros/cabeleireiras nos salões de beleza, a fim de explicar sobre a importância do levantamento de dados, razão para que os profissionais possam ser representados por classe ou entidade trabalhista no município. Aplicou-se o questionário acerca das características sociodemográficas (gênero, idade, escolaridade, raça/cor, estado civil, renda familiar, religião), práticas do consumo de bebidas alcoólicas (consumidores ou não, tempo de consumo, frequência do consumo, tipo de bebida, número de doses) e da identificação do uso em binge drinking: o instrumento denominado questão-chave desta pesquisa. A abordagem ocorreu de forma cautelosa e discreta, em espaços reservados, dentro dos salões, a fim de que fossem assegurados a privacidade e o sigilo das respostas dadas.

A questão-chave foi direcionada a buscar a quantidade de vezes de uso em binge pelos cabeleireiros no último ano. A questão-chave foi proposta pela primeira vez no final da década de 1990 e tinha por pergunta base Have you ever had drinking problem? ${ }^{(7)}$, que, em tradução direta, seria: "Você já teve problemas com bebidas alcóolicas?". Traduzida e validada em estudo realizado no Brasil, em 2017, que denominou a single question como questão-chave, cuja versão final, em português, após a adaptação e a validação cultural, denotou: "Quantas vezes, nos últimos 12 meses, você bebeu X doses ou mais de bebida alcóolica em um dia?". Explica-se, ainda, que esta pergunta apresentou concordância satisfatória entre os juízes em relação ao conteúdo e ao desempenho psicométrico ${ }^{(8)}$. É um instrumento prático, com validade de critério e estratégia apta para ser utilizada no segmento da Atenção Primária em Saúde brasileira para o rastreio do uso de risco de álcool ${ }^{(9)}$.

Os dados foram tabulados em planilhas do programa Microsoft Excel ${ }^{\circledR}$. A análise estatística consistiu na descrição da amostra em frequências absoluta e relativa. Para a análise de normalidade das amostras, aplicou-se o teste de Kolmogorov-Smirnov. Para a avaliação da homogeneidade das variâncias, adotou-se o teste de Levene e, na análise inferencial, utilizaram-se o teste U de Mann-Whitney, para as duas amostras independentes, e o teste de Kruskal Wallis, para a comparação de três ou mais grupos. Diante de rejeição da hipótese nula para o teste de Kruskal Wallis, aplicou-se o teste de Dunn. Para a correlação das variáveis sociodemográficas com o binge, aplicou-se o teste de correlação de Spearman.

Esta pesquisa foi realizada cumprindo integralmente as diretrizes e os trâmites necessários de pesquisas que envolvem seres humanos, obtendo a aprovação do Comitê de Ética e Pesquisa da Universidade Federal do Amapá conforme o Parecer no 2.682.565/2018 e Certificado de Apresentação para Apreciação Ética no 87074418.9.0000.0003. 


\section{Resultados}

Entre os 51 participantes deste estudo, a maioria era do gênero masculino (51,0\%), 68,6\% tinham entre 21 e 40 anos, com Ensino Médio (49,0\%), e $84,3 \%$ eram pardos ou negros. No tocante ao estado civil e à religião, $41,2 \%$ eram solteiros e $49,9 \%$ declararam-se evangélicos. Aponta-se, para as análises subsequentes, que nove participantes $(17,6 \%)$ foram considerados, neste estudo, como não praticantes de uma religião. A renda familiar mensal mais atingida pela maioria dos cabeleireiros incluídos neste estudo, 21 (41,2\%), foi de até $\mathrm{R} \$ 1.164,00$.

Os relatos sobre os dados de consumo alcoólico evidenciaram que 43 (84,3\%) eram consumidores de bebidas alcoólicas e, destes, 26 (51,0\%) possuíam tempo de consumo de um a dez anos. De forma geral, a frequência mais relatada foi raramente, por 20 (39,2\%) participantes, seguida da frequência semanalmente, por 19 (37,3\%) indivíduos. Dentre as bebidas mais ingeridas, sete foram as mais citadas pelos participantes do estudo, sendo a cerveja a mais mencionada (72,5\%). Acerca do número de doses, seis $(11,8 \%)$, no caso das mulheres, bebiam a quantidade permissível e, de forma alarmante, 21 (41,2\%) participantes consumiam mais de 20 doses na mesma ocasião (Tabela 1).

No que concerne à prática do binge drinking entre cabeleireiros, no gênero feminino, constatou-se que $24,0 \%$ não bebiam, $16,0 \%$ faziam uso em binge de uma a três vezes ao ano e 8,0\%, mais de 15 vezes ao ano. No que tange ao gênero masculino, 7,7\% não bebiam, ninguém fazia uso em binge de uma a três vezes ano e 65,5\%, mais de 15 vezes ao ano. Referente à amostra total, comprovou-se que $15,7 \%$ não bebiam, $7,8 \%$ faziam uso em binge de uma a três vezes ao ano e $37,3 \%$, mais de 15 vezes ao ano.
Tabela 1 - Caracterização do consumo de álcool dos cabeleireiros. Oiapoque, AP, Brasil, 2019

\begin{tabular}{|c|c|c|c|}
\hline \multirow[b]{2}{*}{ Variáveis } & \multicolumn{3}{|c|}{ Gênero } \\
\hline & $\begin{array}{c}\text { Masculino } \\
\text { n (\%) }\end{array}$ & $\begin{array}{c}\text { Feminino } \\
\text { n (\%) }\end{array}$ & $\begin{array}{r}\text { Total } \\
\text { n (\%) }\end{array}$ \\
\hline \multicolumn{4}{|c|}{ Consumo de bebidas alcoólicas } \\
\hline Consumidores & $24(92,3)$ & $19(76,0)$ & $43(84,3)$ \\
\hline Não consumidores & $2(7,7)$ & $6(24,0)$ & $8(15,7)$ \\
\hline \multicolumn{4}{|c|}{ Tempo de consumidor de álcool (anos) } \\
\hline $1-5$ & $9(34,6)$ & $7(28,0)$ & $16(31,4)$ \\
\hline $6-10$ & $8(30,8)$ & $2(8,0)$ & $10(19,6)$ \\
\hline $11-15$ & $4(15,4)$ & $5(20,0)$ & $9(17,6)$ \\
\hline $16-20$ & $2(7,7)$ & $3(12,0)$ & $5(9,8)$ \\
\hline $21-25$ & $1(3,8)$ & $1(4,0)$ & $2(3,9)$ \\
\hline$>25$ & - & $1(4,0)$ & $1(2,0)$ \\
\hline \multicolumn{4}{|c|}{ Frequência de consumo de álcool } \\
\hline Raramente & $7(26,9)$ & $13(52,0)$ & $20(39,2)$ \\
\hline Uma vez por mês & $1(3,8)$ & $1(4,0)$ & $2(3,9)$ \\
\hline Semanalmente & $14(53,8)$ & $5(20,0)$ & $19(37,3)$ \\
\hline Quase diariamente & $2(7,7)$ & - & $2(3,9)$ \\
\hline \multicolumn{4}{|c|}{ Tipo de bebida mais consumida } \\
\hline Cerveja & $23(88,5)$ & $14(56,0)$ & $37(72,5)$ \\
\hline Vinho & $16(61,5)$ & $11(44,0)$ & $27(52,9)$ \\
\hline Cachaça & $12(46,2)$ & $9(36,0)$ & $21(41,2)$ \\
\hline Whisky & $14(53,8)$ & $4(16,0)$ & $18(35,3)$ \\
\hline Vodca & $13(50,0)$ & $5(20,0)$ & $18(35,3)$ \\
\hline Caxiri ou caxixi* & $2(7,7)$ & - & $2(3,9)$ \\
\hline Bebida ice com gás & $10(38,5)$ & $10(40,0)$ & $20(39,2)$ \\
\hline Outro tipo de bebida & $6(23,1)$ & - & $6(11,8)$ \\
\hline \multicolumn{4}{|c|}{ Número de doses toda vez que bebem } \\
\hline Não consomem (dose) & $2(7,7)$ & $6(24,0)$ & $8(15,7)$ \\
\hline $1-4$ & - & $6(24,0)$ & $6(11,8)$ \\
\hline $5-9$ & $1(3,8)$ & $2(8,0)$ & $3(5,9)$ \\
\hline $10-14$ & $3(11,5)$ & $6(24,0)$ & $9(17,6)$ \\
\hline $15-19$ & $2(7,7)$ & $2(8,0)$ & $4(7,8)$ \\
\hline$>20$ & $18(69,2)$ & $3(12,0)$ & $21(41,2)$ \\
\hline
\end{tabular}


Na Tabela 2, observam-se diferenças estatísticas nas medianas do uso em binge entre os gêneros, sendo que foi de 17,0 para os homens e de 4,0 para as mulheres, o que sugere que o consumo por parte do gênero masculino foi consideravelmente superior ao do gênero feminino $(p \leq 0,001)$. Quando analisado se o estado civil apresentava alguma relação com o binge, observou-se que a mediana de frequência de ingesta de álcool dos participantes que não tinham parceiros $(15,0)$ era estatisticamente significativa e superior à mediana de vezes consumidas no ano anterior pelos participantes que tinham parceiro (a) $(8,0)$.

A análise de correlação foi procedida entre as variáveis idade, tempo de desempenho na categoria de cabeleireiro e novamente a renda familiar (dessa vez, relacionando os valores de renda de forma crescente). Com relação ao binge constatou-se a correlação $(p=0,025)$ entre a idade e o número de vezes que, no ano anterior, os homens consumiram cinco doses e as mulheres, quatro ou mais doses de bebida alcoólica no mesmo dia. 0 valor de rô de Spearman negativo obtido $(-0,314)$ indicou a correlação inversa, ou seja, com o aumento de idade, o consumo diminui, assim, os cabeleireiros mais jovens apresentaram maior tendência ao consumo do que aqueles com idades mais avançadas (Tabela 2).

O teste de Kruskal-Wallis (K-W), com teste de Dunn como post hoc, foi utilizado para determinar o comportamento do binge, considerando a variável religião, em que se estabeleceram três tratamentos: evangélicos, católicos e uma categoria constituída por "não praticantes" mais um ateu. Assim, não existiram diferenças estatísticas entre a mediana do binge dos católicos $(14,5)$, quando comparada com a mediana dos evangélicos $(10,0)$, sendo a diferença na soma de postos (DSP) determinada pelo post hoc igual a 8,9 e a mediana binge dos não praticantes, incluindo o ateu $(18,0)$, com DSP $=-4,8$. Os valores das medianas do binge de evangélicos foram os mais baixos, sugerindo que estes eram os que menos consumiam álcool e que diferiam estatisticamente $(\mathrm{p}=0,023$ e $\mathrm{DSP}=-13,8$ ) da mediana dos não praticantes e ateus, que apresentavam o maior valor, propondo que estes últimos possuíam maior consumo.

Tabela 2 - Análise estatística da relação entre as variáveis sociodemográficas e a questão-chave. Oiapoque, AP, Brasil, 2019

\begin{tabular}{|c|c|c|c|}
\hline Variáveis & Mediana & Teste estatístico & $\mathbf{p}$ \\
\hline \multicolumn{4}{|l|}{ Gênero } \\
\hline Masculino & $17,0^{*}$ & $U=108,0$ & $\leq 0,001$ \\
\hline Feminino & 4,0 & & \\
\hline \multicolumn{4}{|l|}{ Escolaridade } \\
\hline Ensino Básico & 14,0 & $\mathrm{U}=279,5$ & 0,638 \\
\hline Médio e Superior & 12,0 & & \\
\hline \multicolumn{4}{|l|}{ Estado civil } \\
\hline Com parceiro(a) & $8,0^{*}$ & $U=3204,5$ & 0,024 \\
\hline Sem parceiro(a) & 15,0 & & \\
\hline \multicolumn{4}{|l|}{ Renda familiar } \\
\hline Menor & 13,0 & $U=309,5$ & 0,863 \\
\hline Maior & 12,0 & & \\
\hline \multicolumn{4}{|l|}{ Religião } \\
\hline Evangélicos & $10,0^{\mathrm{a}}$ & $\mathrm{K}-\mathrm{W}=7,547$ & 0,023 \\
\hline Católicos & $14,5^{\mathrm{a}, \mathrm{b}}$ & & \\
\hline Não praticantes e o ateu & $18,0^{\mathrm{b}}$ & & \\
\hline \multicolumn{4}{|l|}{ Correlação com o binge } \\
\hline Idade & 29,0 & Rô $=-0,314$ & 0,025 \\
\hline Tempo na categoria & 9,0 & Rô $=-0,133$ & 0,352 \\
\hline Renda familiar & $4.076,00$ & Rô $=-0,005$ & 0,971 \\
\hline
\end{tabular}

\section{Discussão}

Apesar de possuir uma amostra diminuta, sendo assim a maior limitação deste inquérito, todavia, teve significância, uma vez que coletou as características sociodemográficas, bem como descreveu o consumo de bebidas alcoólicas e a prática do binge drinking entre cabeleireiros. Observou-se que essas variáveis corroboram outros estudos previamente realizados, entretanto, pela ausência de estudos com cabeleireiros, a análise comparativa foi realizada com a população geral ou outros nichos populacionais, com base na 
literatura preexistente.

Desse modo, esta pesquisa é importante no campo da Enfermagem, saúde mental e saúde pública, no intuito de fomentar ações e estratégias que previnam o uso abusivo de álcool, mesmo sendo um consumo ocasional de risco, a fim de reduzir a prática e evitar futuros consumos problemáticos que possam resultar em dependência alcoólica. Além disso, fomenta a prática da Enfermagem dentro do contexto das adições, tendo papel importante na detecção, monitoramento e encaminhamentos necessários diante de pessoas com o uso problemático de álcool, a fim de reduzir o consumo de risco/nocivo, evitando morbidades e problemas relacionados em longo prazo, pois, neste estudo, se identificou alto índice do consumo de bebidas alcóolicas e da prática de binge drinking em cabeleireiros, além do que os maiores índices de binge drinking estavam relacionados ao gênero masculino, aos solteiros e jovens, e a religião evangélica foi associada a um menor ou nenhum consumo de bebidas alcoólicas.

Dessa maneira, achados que se assemelham com os dados do estudo mostraram que $78,0 \%$ dos trabalhadores consumiram bebidas alcoólicas nos últimos 12 meses, 82,0\% dos trabalhadores públicos de manutenção faziam uso em binge, os homens faziam consumo de álcool com maior frequência $(63,0 \%$ uso em binge) e 44,0\%, o consumo abusivo ou provável dependência ${ }^{(10)}$. Pesquisa com estudantes brasileiros acerca do beber pesado episódico apontou que 31,7\% dos homens, em comparação a 19,5\% das mulheres, praticavam o uso em binge, sendo a cerveja a bebida mais utilizada ${ }^{(11)}$.

Ainda em se tratando do uso de álcool entre os estudantes, 89,2\% afirmaram o consumo nos últimos três meses pesquisados e 51,6\% afirmaram o uso em binge, sendo essa prática associada a maiores chances de problemas e consequências advindos do uso de bebidas alcóolicas ${ }^{(12)}$. Outro inquérito, com 182 estudantes de Enfermagem, mostrou que 48,9\% praticaram uso em binge, sendo associadas a frequência e a dose de consumo ${ }^{(13)}$.

Em se tratando das variáveis que deram asso- ciação, estudo, que analisou os fatores sociodemográficos associados ao uso de álcool, segundo o gênero, em território brasileiro, mostrou que a maior frequência do uso episódico excessivo de álcool foi de 13,7\%, sendo $21,6 \%$ entre os homens e $6,6 \%$ entre as mulheres, com maior prevalência em pessoas entre $25 \mathrm{e}$ 34 anos e solteiras, dados concordantes com os desta pesquisa ${ }^{(14)}$. Resultados acerca do perfil do consumo de álcool e do uso ocasional de risco entre estudantes universitários mostrou que 51,6\% faziam uso em bin$g e$, sendo associados ao gênero masculino e ser solteiros, resultados que corroboram o público investigado neste inquérito ${ }^{(15)}$.

Desse modo, constatou-se a existência do consumo de álcool entre o público jovem a partir dos estudos elencados. Especula-se que o consumo de álcool e a prática de binge drinking são maiores entre os homens, por questões culturais e ancestralidade, nos quais o consumo foi mais facilmente aceito e, em se tratando do consumo de risco, isso se torna mais evidente, até mesmo por questões de masculinidade, apresentando prática de consumo de álcool maior do que entre as mulheres. Quanto aos solteiros e às pessoas jovens, isto se deve à liberdade, ao fato de reunir-se com amigos, frequentar festas e locais, com o intuito de lazer e diversão, com mais frequência e, assim, tornando-se mais vulneráveis ao consumo de risco, dados refletidos a partir dos estudos relacionados.

Ao proceder ao comparativo do consumo de álcool com outros nichos populacionais, no município de Oiapoque, local este que se destaca pela vulnerabilidade social e econômica, uma pesquisa com mototaxistas, que observou uma tendência entre a naturalidade e o consumo de risco, apontou que $48,5 \%$ consumiam bebidas alcóolicas, sendo que $16,2 \%$ faziam uso de risco, $7,4 \%$, uso nocivo e 1,5\%, provável dependência, e a quantidade de doses maior que cinco doses, na mesma ocasião, aumentava em 20 vezes a chance de desencadear um consumo problemático ${ }^{(16)}$.

Outro inquérito, também realizado em Oiapoque com servidores de uma instituição de ensino, mostrou que $24,0 \%$ dos trabalhadores faziam uso de risco e $2,0 \%$, consumo nocivo, além de pontuar condi- 
ções de trabalhos inadequadas, campus em construção, que a maioria dos servidores é de outros Estados, mergulhando em outras realidade de vida, falta de opções de lazer, sendo a bebida a principal fonte de diversão e, assim, um fator de risco para o consumo problemático ${ }^{(17)}$.

Investigação realizada com motoristas de transporte alternativo, na fronteira franco-brasileira, apresentou que $100,0 \%$ dos entrevistados consumiam bebidas alcoólicas, sendo que 17,9\% faziam o uso ocasional, $53,8 \%$, o uso abusivo e $28,2 \%$ tinham indicativo de provável dependência, apontando, assim, a vulnerabilidade dos motoristas e refletindo, assim, a necessidade de instigar os profissionais de saúde, dentre eles, os enfermeiros, a desenvolverem ações educativas com o intuito de redução do consumo, morbimortalidades e acidentes causados pelo uso de álcool ${ }^{(18)}$.

Uma variável que apresentou relação estatística e merece reflexão foi a religião, mostrando que ser evangélico tem menos chances de consumo de álcool, sendo, portanto, fator protetor, estando a religiosidade associada ao não consumo em binge, ou seja, a prática da religiosidade favorece uma postura de autocontrole quanto ao uso de álcool. Porém, estudos são preliminares e há a necessidade de mais pesquisas com melhor sedimentação e esclarecimentos dessas associações $^{(19)}$.

Por fim, a espiritualidade/religiosidade mostra-se como variável relevante no âmbito da drogadição, sendo fator preventivo, protetivo e curativo, mostrando evidências científicas em uma relação mais positiva do que negativa, apontando, ainda, a necessidade de mais estudos nesse contexto, focando em estudos longitudinais e no aprofundamento da temática para maiores subsídios ${ }^{(20)}$.

Portanto, pautada nos danos causados pelo consumo abusivo de álcool, no âmbito geral, sobreleva-se a importância da elaboração de estratégias educacionais, orientações e esclarecimentos acerca dos prejuízos ocasionados pelo uso de bebidas alcóolicas $^{(16-18)}$, mesmo aos indivíduos que não fazem uso em binge, para que, assim, se possam estimular dis- cussões juntos aos órgãos competentes pela disseminação e formação do conhecimento junto a essa classe trabalhadora, impossibilitando, dessa maneira, o adoecimento e o surgimento de casos que resultem na dependência alcoólica, destacando-se, nesse contexto, a Enfermagem, que ocupa uma posição privilegiada no processo de detecção precoce, promoção de saúde, prevenção de agravos e medidas de reabilitação.

\section{Conclusão}

O consumo de álcool entre cabeleireiros foi considerado alto e a prática de binge drinking, frequente, ou seja, os que consumiam bebidas alcóolicas faziam uso ocasional de risco, dado preocupante e, possivelmente, condição para a provável dependência alcoólica. 0 risco de consumo elevado de álcool foi relacionado com o gênero masculino, ser solteiro ou não ter parceiro e ser jovem. 0 fato de pertencer à religião evangélica foi associado a um menor ou nenhum consumo de bebidas alcoólicas.

\section{Colaborações}

Castelo Branco FMF contribuiu na análise e interpretação dos dados, redação do artigo, revisão crítica relevante do conteúdo intelectual e aprovação final da versão a ser publicada. Sousa GT, Marques NQS, Moraes AAS e Dutok-Sánchez CM contribuíram na concepção do projeto, coleta e análise dos dados e redação do artigo. Trindade FR e Branco Neto TC contribuíram na interpretação dos dados, revisão crítica relevante do conteúdo intelectual e aprovação final da versão a ser publicada.

\section{Referências}

1. National Institute on Alcohol and Alcoholism. Rethinking drinking [Internet]. 2019 [cited Ago. 26, 2020]. Available from: https://www.rethinkingdrinking.niaaa.nih.gov/

2. World Health Organization. Global report on alcohol [Internet]. 2018 [cited Mar. 17, 2020]. Available from: https://apps.who.int/iris/rest/ bitstreams $/ 1151838 /$ retrieve 
3. Munhoz TN, Santos IS, Nunes BP, Mola CL, Silva ICM, Matijasevich A. Tendências de consumo abusivo de álcool nas capitais brasileiras entre os anos de 2006 a 2013: análise das informações do VIGITEL. Cad Saúde Pública 2017; 33(7):e00104516. doi: https://doi. org/10.1590/0102311x00104516

4. Bastos FIPM, Vasconcellos MTL, Boni RB, Reis NB, Coutinho CFS. III Levantamento Nacional sobre o uso de drogas pela população brasileira. Rio de Janeiro: FIOCRUZ/ICICT; 2017.

5. Bordin V, Alves DCI, Martins LK, Luz MS, Matos FGOA. Reprocessing of materials used in beauty salons and biosafety of professionals involved. J Health NPEPS [Internet]. 2018 [cited Ago 26, 2020];3(2):441-56. Available from: http://docs. bvsalud.org/biblioref/2019/02/980870/310611078-1-pb.pdf

6. Barbiani R, Dalla Nora CR, Schaefer R. Nursing practices in the primary health care context: a scoping review. Rev Latino-Am Enfermagem. 2016; 24:e2721. doi: http://dx.doi.org/10.1590/15188345.0880.2721

7. Fleming MF, Barry KL, Manwell LB, Jhon K, London R. Brief physician advice for problem alcohol drinkers: a randomized controlled trial in community-based primary care practices. JAMA. 1997; 277(13):1039-45. doi: https://doi. org/10.1001/jama.1997.03540370029032

8. Maciel MED, Vargas D. Cultural adaptation and content validation of the Single-Question for screening alcohol abuse. Rev Esc Enferm. USP. 2017; 41:e03292. doi: https://doi.org/10.1590/ s1980-220x2016048703292

9. Maciel MED, Vargas D. Criterion validity of the Key Question for screening at-risk alcohol use in primary healthcare. Rev Esc Enferm USP. 2020; 54:e03553. doi: http://dx.doi.org/10.1590/ S1980-220X2018032503553

10. Oliveira JL, Souza J. Factors associated with alcohol consumption among public maintenance workers. Acta Paul Enferm. 2018; 31(1):17-24. doi: https:// doi.org/10.1590/1982-0194201800004

11. Espíndola MI, Schneider DR, Bartillot CB. The perception of college students about the consequences of binge drinking. Rev Elet Saúde Mental Álcool Drogas. 2019; 15(2):29-37. doi:https:// dx.doi.org/10.11606/issn.1806-6976. smad.2019.149204
12. Bedendo A, Andrade ALM, Opaleye ES, Noto AR. Binge drinking: a pattern associated with a risk of problems of alcohol use among university students. Rev Latino-Am Enfermagem. 2017; 25:e2925. doi: https://dx.doi.org/10.1590/15188345.1891.2925

13. Sousa BOP, Souza ALT, Cubas Junior D, Silva GF, Santos MA, Pillon SC. Association between binge drinking and smoking in nursing students. Rev Eletr Enf. 2018; 20:v20a36. doi: https://doi. org/10.5216/ree.v20.48611

14. Machado IE, Monteiro MG, Malta DC, Lana FCF. Brazilian health survey (2013): relation between alcohol use and sociodemographic characteristics by sex in Brazil. Rev Bras Epidemiol. 2017; 20(3):408-22. doi: https://doi. org/10.1590/1980-5497201700030005

15. Pelicioli M, Barelli C, Gonçalves CBC, Hahn SR, Scherer JI. Alcohol consumption and episodic heavy drinking among undergraduate students from the health area of a Brazilian university. J Bras Psiquiatr. 2017; 66(3):150-6. doi: https:// doi.org/10.1590/0047-2085000000164

16. Silva JM, Silva RA, Castelo Branco FMF. Pattern of alcohol consumption among motorcycle taxi drivers. Rev Rene. 2020; 21:e43603. doi: https:// doi.org/10.15253/2175-6783.20202143603

17. Castelo Branco FMF, Faria FLS, Dutok CMS, Branco Neto TC, Vargas D, Barroso TMMDA. The patterns of consumption of alcohol among employees of a Brazilian public university. Rev Ref. 2019; IV(22). doi: https://dx.doi.org/10.12707/RIV19026

18. Branco F, Mamede L, Neto T, Barroso TM. Pattern of consumption of psychoactive substances in alternative transport drivers. Millenium. 2020; 2(12):69-77. doi: 10.29352/mill0212.06.00303

19. Guimarães MO, Paiva PCP, Paiva HN, Lamounier JA, Ferreira EF, Zarzar PMPA. Religiosidade como possível fator de proteção do "binge drinking" por escolares de 12 anos de idade: um estudo de base populacional. Ciênc Saúde Coletiva. 2018; 23(4):1067-76. doi: https://doi. org/10.1590/1413-81232018234.04872016

20. Esperandio MRG, Côrrea MR. O papel da espiritualidade/religiosidade no fenômeno da drogadicção: uma revisão integrativa de literatura. Rev Estd Religião. 2017; 17(2):73-98. doi: https:// doi.org/10.23925/1677-1222.2017vol17i2a5

\section{(cc) BY}

Este é um artigo de acesso aberto distribuído sob os termos da Licença Creative Commons 